\title{
Association of Complement C3 with Clinical Deterioration Among Hospitalized Patients with COVID-19
}

\author{
Hongbo Jiang ${ }^{1} *$, Qiaosen Chen ${ }^{1} *$, Shaoping Zheng ${ }^{2}$, Chunxia Guo ${ }^{3}$, Jinzhuo Luo ${ }^{3}$, Hua Wang ${ }^{3}$, \\ Xin Zheng ${ }^{3}$, Zhihong Weng ${ }^{3}$ \\ 'Department of Epidemiology and Biostatistics, School of Public Health, Guangdong Pharmaceutical University, Guangzhou, People's Republic of \\ China; ${ }^{2}$ Department of Ultrasound, Union Hospital, Tongji Medical College, Huazhong University of Science and Technology, Wuhan, People's \\ Republic of China; ${ }^{3}$ Department of Infectious Diseases, Union Hospital, Tongji Medical College, Huazhong University of Science and Technology, \\ Wuhan, People's Republic of China
}

*These authors contributed equally to this work

Correspondence: Zhihong Weng, Department of Infectious Diseases, Union Hospital, Tongji Medical College, Huazhong University of Science and Technology, 1277 JieFang Avenue, Wuhan, 430022, People's Republic of China, Email wengzh@hust.edu.cn

Background: The role of the complement system in coronavirus disease 2019 (COVID-19) remains controversial. This study aimed to evaluate the relationship between serum complement $\mathrm{C} 3$ levels, clinical worsening, and risk of death in hospitalized patients with COVID-19.

Methods: Data were collected from 216 adults with COVID-19 admitted to a designated clinical center in Wuhan Union Hospital (China) between February 13, 2020, and February 29, 2020. Their complement C3 levels were measured within 24 h of admission. The primary outcome was a clinical worsening of 2 points on a 6-point ordinal scale. The secondary outcome was all-causes of death. Inverse probability of treatment weighting (IPTW) analysis was conducted to adjust for the baseline confounders.

Results: The median value of $\mathrm{C} 3$ was 0.89 (interquartile range, $0.78-1.01) \mathrm{g} / \mathrm{L}$. Clinical worsening occurred in $12.3 \%$ (7/57) and 2.5\% (4/159) of patients with baseline $\mathrm{C} 3$ levels $<$ and $\geq 0.79 \mathrm{~g} / \mathrm{L}$, respectively (hazard ratio [HR], 5.22; 95\% confidence interval [CI], 1.53-17.86). After IPTW adjustment, the risk for clinical worsening was 4-fold greater (weighted HR, 4.61; 95\% CI, 1.16-18.4) in patients with C3 levels less than $0.79 \mathrm{~g} / \mathrm{L}$ comparatively. The sensitivity analyses revealed the robustness of the results. No significant associations between $\mathrm{C} 3$ levels and death were observed on unadjusted (HR, 2.92; 95\% CI, 0.73-11.69) and IPTW analyses (weighted HR, 3.78; 95\% CI, 0.84-17.04).

Conclusion: Low complement C3 levels are associated with a higher risk for clinical worsening among inpatients with COVID-19. The serum C3 levels may contribute to the identification of patient populations that could benefit from therapeutic complement inhibition.

Keywords: SARS-CoV-2, COVID-19, complement C3, clinical worsening

\section{Introduction}

The coronavirus disease 2019 (COVID-19), which is caused by the severe acute respiratory syndrome coronavirus 2 (SARS-CoV-2), has resulted in a global pandemic and considerable mortality. The fatal outcomes from COVID-19 are attributed mainly to an overactive immune response to the SARS-CoV-2 infection. ${ }^{1}$ However, hyperinflammation in patients with COVID-19 remains poorly clarified.

Complement, a critical component of the innate immune system, plays a key role against various pathogens, including bacteria and viruses. However, dysregulated activation of the complement can also lead to severe diseases like thrombotic microangiopathy. ${ }^{2}$ It was indicated that complement activation is associated with the pathogenesis of the Middle East respiratory syndrome (MERS) ${ }^{3,4}$ and severe acute respiratory syndrome (SARS), ${ }^{5}$ which are caused by coronaviruses similar to SARS-CoV-2. A previous study used a mouse model to demonstrate that complement activation 
occurred in response to SARS-CoV infection, and mice deficient in C3, a central complement component, had less systemic inflammation. ${ }^{5}$

Evidence from recent studies suggests that complement activation may help the pathogenesis of SARS-CoV-2 infection by triggering a severe cytokine storm. ${ }^{6,7}$ However, studies on the role of the complement system in COVID19 are limited by controversial results. A study showed that low complement $\mathrm{C} 3$ levels were associated with poor prognosis in COVID-19 patients. $^{8}$ However, Qin et al reported that there were no significant differences in the levels of C3 between the mild and severe groups. ${ }^{9}$ Moreover, other data showed that C3 levels cannot predict COVID-19 progression. ${ }^{10}$ In this study, our goal was to investigate whether complement $\mathrm{C} 3$ level is associated with the risk for clinical worsening or death among inpatients with COVID-19.

\section{Materials and Methods}

\section{Study Design}

In this retrospective cohort study, we collected data from patients with laboratory-confirmed COVID-19 in the Tumor Center, which is a designated clinical center for COVID-19 in the Union Hospital, Tongji Medical College of Huazhong University of Science and Technology (Wuhan, China), between February 13, 2020, and February 29, 2020. We screened patients aged $\geq 18$ y with serum complement C3 levels measured within $24 \mathrm{~h}$ of admission. Definite outcomes (discharge or death) were followed up until March 15, 2020.

Patients included in this study were diagnosed using the guidelines of the Diagnosis and Treatment of Novel Coronavirus Pneumonia issued by the Chinese National Health Commission. ${ }^{11}$ Data on demographic characteristics, clinical features, underlying diseases, laboratory examinations, treatments, and clinical outcomes were extracted from the patients' electronic medical records. Two trained physicians (CXG and JZL) evaluated them independently. This study was approved by the Ethics Commission of Tongji Medical College of Huazhong University of Science and Technology in Wuhan (2020-0058). Written informed consent was waived because of the rapidly emerging infectious disease and requirements for epidemic prevention and control. The patients' personal information was de-identified to ensure confidentiality and comply with the Declaration of Helsinki.

\section{Exposure}

The normal values of serum C3 levels may vary slightly among different laboratories. The normal value ranged from 0.80 to $1.78 \mathrm{~g} / \mathrm{L},{ }^{12} 0.88$ to $2.01 \mathrm{~g} / \mathrm{L},{ }^{13}$ or 0.80 to $1.60 \mathrm{~g} / \mathrm{L}$. ${ }^{14}$ Some laboratories showed normal values ranging between 0.88 and $2.52 \mathrm{~g} / \mathrm{L}$ and 0.88 and $2.06 \mathrm{~g} / \mathrm{L}$ for men and women, respectively. ${ }^{15} \mathrm{In}$ our hospital, the reference range of serum complement $\mathrm{C} 3$ levels was $0.79-1.52 \mathrm{~g} / \mathrm{L}$ for both men and women. The participants were divided into two groups based on their serum complement C3 levels tested at baseline. Patients with $\mathrm{C} 3$ levels less than $0.79 \mathrm{~g} / \mathrm{L}$ were in the low C3 group and those with $\mathrm{C} 3$ levels of $0.79 \mathrm{~g} / \mathrm{L}$ or greater were in the control group. The study baseline for each patient was $24 \mathrm{~h}$ after hospital admission.

\section{Covariates}

Potential confounding factors were chosen according to previously published articles and physician advice, including demographics (age and gender), comorbidities (hypertension, coronary heart disease [CHD], and diabetes), laboratory findings (neutrophils, lymphocytes, C-reactive protein [CRP], and D-dimer), and medications (arbidol, interferon $\alpha-2 b$, ribavirin, antibiotics, low molecular weight heparin, and Chinese medicine).

\section{Outcomes}

The primary outcome was clinical worsening, which was defined as all-causes of death during the hospital stay, or an increase of at least 2 points from baseline on the 6-point ordinal scale, ${ }^{16}$ whichever came first. The 6-point ordinal scale was as follows: 1 = discharge; 2 = hospitalized without supplemental oxygen; 3 = hospitalized, requiring low-flow oxygen; 4 = hospitalized, requiring noninvasive mechanical ventilation or high-flow oxygen; $5=$ hospitalized, requiring 
invasive ventilation or extracorporeal membrane oxygenation; and $6=$ death. ${ }^{16}$ The secondary outcome was only allcauses of death.

\section{Statistical Analysis}

Categorized variables were summarized using frequency and proportion. Continuous variables were summarized using the mean and standard deviation. We used absolute standardized mean difference (aSMD) for evaluating the balance between the low $\mathrm{C} 3$ and control groups.

We conducted the univariate analysis to establish the relationship between low C3 and clinical worsening. In observational studies, univariate analysis is not reliable enough. Therefore, inverse probability treatment weighting (IPTW) was applied for adjusting variables to keep them comparable between the two groups. ${ }^{17}$ Meanwhile, two survival curves were generated using the Kaplan-Meier method. One was based on univariate survival analysis and the other was adjusted using IPTW analysis. For examining the robustness of the results, we performed a series of sensitivity analyses: (1) excluding the different combinations of variables that were included in the primary analysis model; (2) trimming the extreme value of weights. Then, we re-conducted the IPTW analysis. Besides clinical worsening, we explored the relationship between overall survival and low C3 with crude and IPTW analyses using the Cox proportional-hazards regression model. The formula for the IPTW modeling is provided in the Supplementary Methods.

Missing data were handled using multiple imputations. Proportional hazards assumption was verified using Schoenfeld residuals test, and no evidence of violation was found. All statistical analyses were performed using the software R version 4.0.2 (https://www.r-project.org/).

\section{Results}

\section{Characteristics of the Patients}

A total of 955 patients with COVID-19 were admitted to this designated clinical center between February 13, 2020, and February 29, 2020. Among them, 728 had no information regarding serum complement C3 levels in their electronic medical records, and 11 were not tested within the $24 \mathrm{~h}$ window. Therefore, 216 patients with C3 levels measured within $24 \mathrm{~h}$ of admission were included in this study (Figure 1). The median value of C3 level was 0.89 (interquartile range [IQR], 0.78-1.01) g/L. Of the 216 patients, 57 (26.4\%) and $159(73.6 \%)$ had C3 levels $<$ and $\geq 0.79$ g/L, respectively. The baseline demographics and clinical characteristics of the low C3 and control groups are summarized in Table 1.

\section{Primary Outcome}

Clinical outcomes of the 216 patients on 15 March 2020, according to the 6-point ordinal scale, are shown in Supplementary Table 1 . In the crude analysis, the cumulative incidences of clinical worsening were $12.3 \%(7 / 57)$ and $2.5 \%$ (4/159) among patients with baseline C3 levels $<$ and $\geq 0.79 \mathrm{~g} / \mathrm{L}$, respectively. Clinical worsening was frequent among patients in the low C3 group compared to the control group (HR, 5.22; 95\% CI, 1.53-17.86) (Figure 2, left panel).

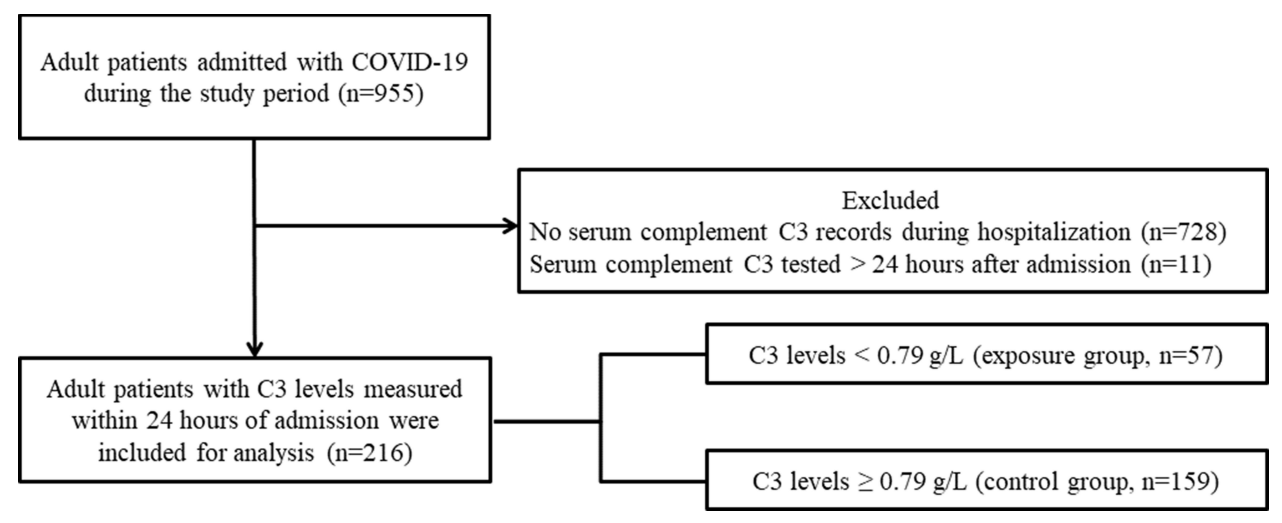

Figure I Flow chart of study participants. The study baseline was defined as $24 \mathrm{~h}$ after admission. COVID-19 denotes coronavirus disease 2019. 
Table I Baseline Characteristics of COVID-19 Patients with Low C3 Levels or Not, Before and After Inverse Probability Treatment Weighting

\begin{tabular}{|c|c|c|c|c|c|c|c|c|}
\hline \multirow[t]{3}{*}{ Variable } & \multicolumn{3}{|c|}{ Before Weighting } & \multirow[t]{3}{*}{ aSMD } & \multicolumn{3}{|c|}{ After Weighting } & \multirow[t]{3}{*}{ aSMD } \\
\hline & Low C3 Group & Control Group & Overall & & Low C3 Group & Control Group & Overall & \\
\hline & $(N=57)$ & $(N=159)$ & $(N=216)$ & & $(N=57)$ & $(N=158)$ & $(N=215)$ & \\
\hline Age, yrs, mean (SD) & $65.67(15.00)$ & $61.19(12.72)$ & $62.38(13.47)$ & 0.33 & $62.57(15.81)$ & $62.12(12.29)$ & $62.24(13.27)$ & 0.03 \\
\hline Gender, n (\%) & - & - & - & 0.01 & - & - & - & 0.03 \\
\hline Male & $26(45.6)$ & $72(45.3)$ & $98(45.4)$ & - & $26.5(46.8)$ & $71.8(45.4)$ & $98.3(45.8)$ & - \\
\hline Female & $31(54.4)$ & 87 (54.7) & $118(54.6)$ & - & $30.1(53.2)$ & $86.2(54.6)$ & II $6.3(54.2)$ & - \\
\hline \multicolumn{9}{|l|}{ Comorbidities } \\
\hline Hypertension, n (\%) & $7.0(12.3)$ & $25.0(15.7)$ & $32.0(14.8)$ & 0.10 & $9.4(16.6)$ & $24.0(15.2)$ & $33.4(15.6)$ & 0.04 \\
\hline Diabetes, n (\%) & $4.0(7.0)$ & $11.0(6.9)$ & $15.0(6.9)$ & $<0.01$ & $3.2(5.6)$ & $10.2(6.5)$ & $13.4(6.2)$ & 0.04 \\
\hline Coronary heart disease, n (\%) & $2.0(3.5)$ & $7.0(4.4)$ & $9.0(4.2)$ & 0.04 & $1.8(3.2)$ & $6.2(3.9)$ & $8.0(3.7)$ & 0.04 \\
\hline \multicolumn{9}{|l|}{ Laboratory tests } \\
\hline Neutrophils $\leq 6.3 \times 10^{\wedge} 9 / \mathrm{L}, \mathrm{n}(\%)$ & $3.0(5.3)$ & $17.0(10.7)$ & $20.0(9.3)$ & 0.19 & $4.9(8.7)$ & $14.6(9.2)$ & $19.5(9.1)$ & 0.02 \\
\hline Lymphocytes $\leq 1.1 \times 10^{\wedge} 9 / \mathrm{L}, \mathrm{n}(\%)$ & $29.0(50.9)$ & $109.0(68.6)$ & 138.0 (63.9) & 0.37 & $33.8(59.7)$ & $101.2(64.1)$ & $135.0(62.9)$ & 0.09 \\
\hline D-dimer $\leq$ Img/L, n (\%) & $16.0(36.4)$ & $38.0(29.5)$ & $54.0(31.2)$ & 0.09 & $13.6(30.4)$ & $38.9(30.2)$ & $52.5(30.2)$ & 0.02 \\
\hline C-reactive protein $\leq 8 \mathrm{mg} / \mathrm{L}, \mathrm{n}(\%)$ & $24.0(42.1)$ & $64.0(40.5)$ & $88.0(40.9)$ & 0.02 & $23.8(42.1)$ & $63.0(40.1)$ & $86.8(40.6)$ & 0.03 \\
\hline \multicolumn{9}{|l|}{ Treatments } \\
\hline LMWH, n (\%) & $47.0(82.5)$ & $141.0(88.7)$ & $188.0(87.0)$ & 0.18 & $50.4(89.0)$ & $139.4(88.2)$ & I 89.7 (88.4) & 0.02 \\
\hline Arbidol, n (\%) & $53.0(93.0)$ & $152.0(95.6)$ & $205.0(94.9)$ & 0.12 & $53.2(94.0)$ & $149.2(94.5)$ & $202.5(94.4)$ & 0.02 \\
\hline Ribavirin, n (\%) & $7.0(12.3)$ & $22.0(13.8)$ & $29.0(13.4)$ & 0.05 & $6.7(11.8)$ & $22.0(13.9)$ & $28.6(13.3)$ & 0.06 \\
\hline Interferon $\alpha-2 b, n(\%)$ & $13.0(22.8)$ & $33.0(20.8)$ & $46.0(21.3)$ & 0.05 & $11.9(21.0)$ & 33.1 (20.9) & $44.9(20.9)$ & $<0.01$ \\
\hline Chinese medicine, n (\%) & $45.0(78.9)$ & $148.0(93.1)$ & $193.0(89.4)$ & 0.46 & $50.6(89.3)$ & 141.7 (89.7) & $192.3(89.6)$ & 0.01 \\
\hline Antibiotics, n (\%) & $44.0(77.2)$ & $120.0(75.5)$ & 164.0 (75.9) & 0.04 & $43.2(76.3)$ & $119.3(75.5)$ & $162.5(75.7)$ & 0.01 \\
\hline
\end{tabular}

Abbreviations: COVID-19, coronavirus disease 2019; aSMD, absolute standardized mean difference; LMWH, low molecular weight heparin.

Similarly, there was a significant difference in the cumulative incidences of clinical worsening between the two groups in the IPTW analysis (weighted HR, 4.61; 95\% CI, 1.16-18.4) (Figure 2, right panel). The distribution of weight and aSMD of variables in the IPTW analysis for clinical worsening are shown in Supplementary Figures 1 and 2, respectively.

\section{Secondary Outcome}

Over a median follow-up of $29.1 \mathrm{~d}$ (IQR, 28.6-31.2 d), the secondary outcome developed in eight (3.7\%) of the 216 patients. Four patients died in both the low C3 (0.081 case/person-month) and control groups ( 0.027 case/person-month). At the end of the follow-up on 15 March 2020, 205 (94.9\%) patients were discharged alive, and three (1.4\%) remained in the hospital.

Figure 3 shows the Kaplan-Meier curves. Both the unadjusted (HR, 2.92; 95\% CI, 0.73-11.69) and IPTW analyses (weighted HR, 3.78; 95\% CI, 0.84-17.04) showed insignificant differences between the two groups. However, they indicated that the low $\mathrm{C} 3$ population might be associated with a higher risk of death.

\section{Sensitivity Analysis}

Sensitivity analysis for the primary outcome was robust under different contexts of the model. Some important variables, including D-dimer, CRP, diabetes, CHD, and hypertension, were excluded intentionally and the conclusion remained unchanged (Table 2). Therefore, it is rational to assume that the bias would have been less even if we neglected these variables in the primary analysis. Moreover, we added the weight-trimming analysis to simulate a scenario where the positivity assumption did not hold up and found that the estimate was still robust (Table 2). 
Strata + Control + Low C3

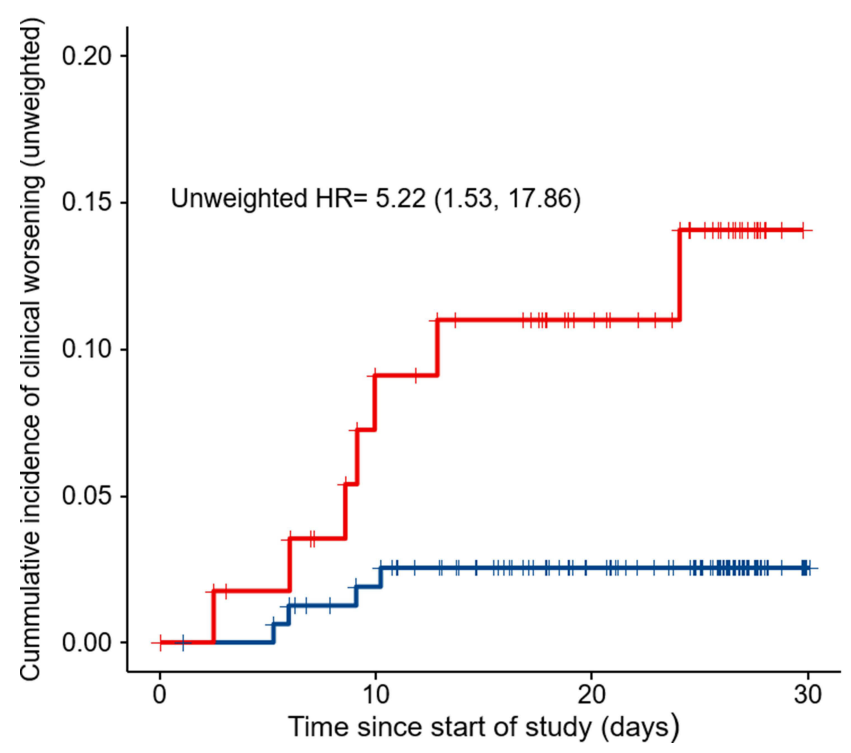

Strata + Control + Low C3

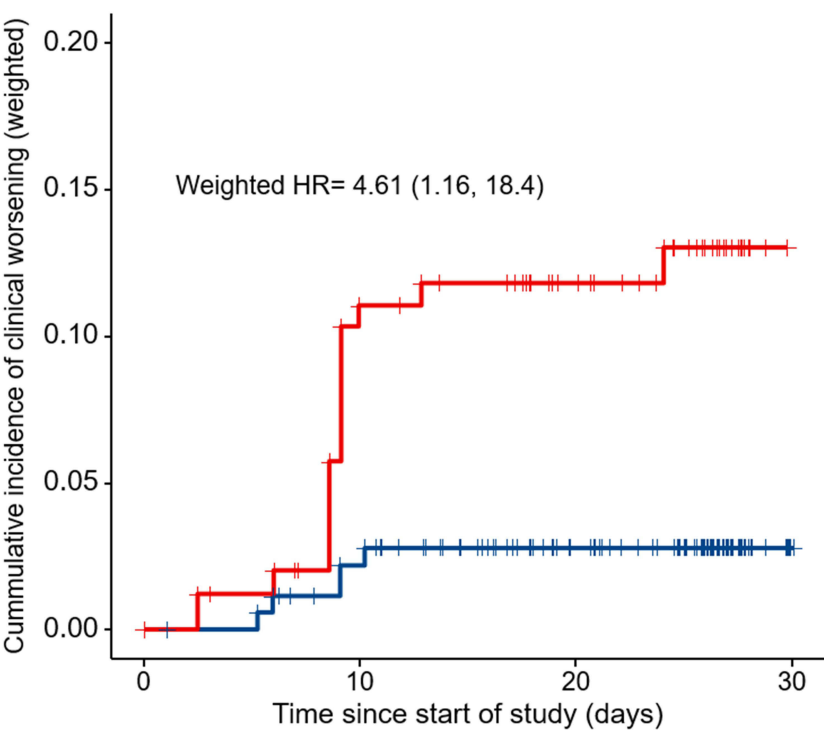

Figure 2 Kaplan-Meier curves for clinical worsening during follow-up, both before and after the inverse probability of treatment weighting (IPTW). There was a significant association between the baseline serum $\mathrm{C} 3$ levels and clinical worsening on unadjusted (crude HR, 5.22; 95\% Cl, I.53-17.86) and IPTW analyses (weighted HR, 4.6I; $95 \%$ $\mathrm{Cl}, 1.16-18.4)$.
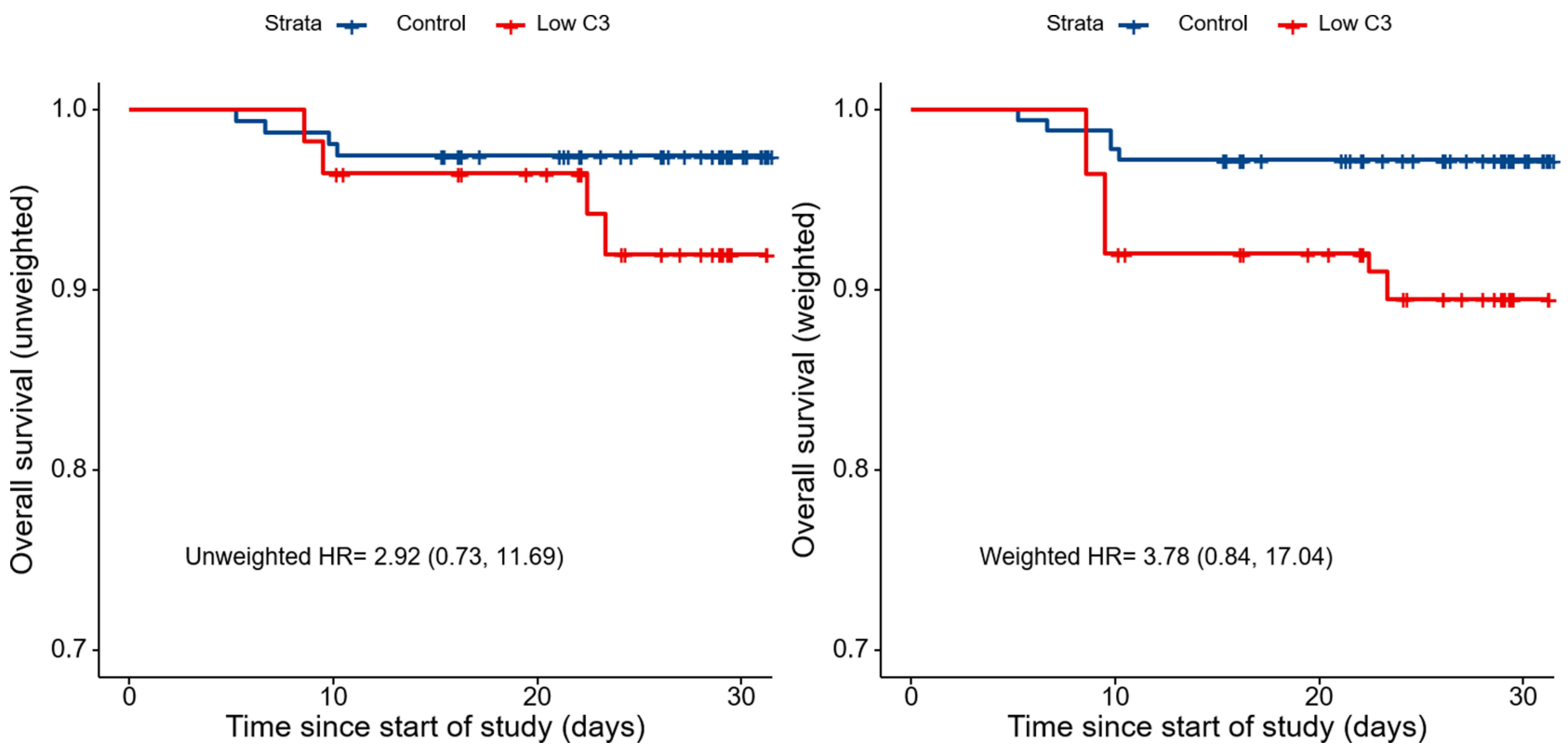

Figure 3 Kaplan-Meier curves for overall survival during follow-up, both before and after the inverse probability of treatment weighting (IPTW). There was no significant association between the baseline serum $\mathrm{C} 3$ levels and death on unadjusted (crude HR, 2.92; 95\% Cl, 0.73-II.69) and IPTW analyses (weighted HR, 3.78; 95\% CI, 0.84-17.04).

\section{Discussion}

It has been hypothesized that complement activation may contribute to the pathogenesis of COVID-19. Our study provides clinical evidence based on real-world data to support such a theoretical perspective. In this observational study, we investigated the relationship between serum complement C3 levels, clinical worsening, and risk of death in a retrospective cohort of 216 hospitalized patients with confirmed COVID-19. Our results suggested that in COVID19 patients who have baseline $\mathrm{C} 3$ levels $<0.79 \mathrm{~g} / \mathrm{L}$, there was a 4-fold greater increase in the risk for clinical worsening 
Table 2 Summarization of Different Analytical Strategies to Explore the Associations of Low C3 with Outcomes of COVID-19 Patients

\begin{tabular}{|l|c|}
\hline Analytical Strategy & HR (95\% Cl) \\
\hline $\begin{array}{l}\text { Clinical worsening (primary analysis) } \\
\text { Crude analysis }\end{array}$ & $5.22(1.53-17.86)$ \\
IPTW analysis & $4.61(1.16-18.40)$ \\
\hline Clinical worsening (sensitivity analysis-ignoring important variable) & \\
D-dimer excluded & $4.52(1.14-17.94)$ \\
D-dimer and C-reactive protein excluded & $4.27(1.08-16.96)$ \\
Diabetes excluded & $4.78(1.21-18.92)$ \\
Diabetes and coronary heart disease excluded & $4.89(1.23-19.43)$ \\
Diabetes, coronary heart disease and hypertension excluded & $4.07(1.02-16.23)$ \\
\hline Clinical worsening (sensitivity analysis-weight-trimming analysis) & \\
$0.5 \%$ and $99.5 \%$ weights trimming & $4.45(1.12-17.58)$ \\
$1.0 \%$ and $99.0 \%$ weights trimming & $4.38(1.11-17.25)$ \\
1.5\% and 98.5\% weights trimming & $4.36(1.11-17.13)$ \\
$2.0 \%$ and $98.0 \%$ weights trimming & $4.35(1.11-17.04)$ \\
$2.5 \%$ and $97.5 \%$ weights trimming & $4.32(1.11-16.84)$ \\
$5.0 \%$ and $95.0 \%$ weights trimming & $4.23(1.13-15.80)$ \\
$7.5 \%$ and $92.5 \%$ weights trimming & $4.35(1.20-15.78)$ \\
$10.0 \%$ and $90.0 \%$ weights trimming & $4.65(1.31-16.45)$ \\
\hline Overall survival (secondary analysis) & \\
Crude analysis & $2.92(0.73-11.69)$ \\
IPTW analysis & $3.78(0.84-17.04)$ \\
\hline
\end{tabular}

Abbreviations: COVID-19, coronavirus disease 2019; HR, hazard ratio; $\mathrm{Cl}$, confidence interval; IPTW, inverse probability of treatment weighting.

when compared to patients with $\mathrm{C} 3$ levels $\geq 0.79 \mathrm{~g} / \mathrm{L}$ (weighted HR, 4.61; 95\% CI, 1.16-18.4). The sensitivity analyses revealed the robustness of the results as well.

The complement system, a bridge between innate and adaptive immunity, can identify and eliminate invading viruses through opsonization, promoting virus-specific immune responses and neutralizing cell-free viruses. ${ }^{18,19}$ After activation, the mature complement $\mathrm{C} 3$ is cleaved to generate $\mathrm{C} 3 \mathrm{a}$ and $\mathrm{C} 3 \mathrm{~b}$. Further cleavage of $\mathrm{C} 3 \mathrm{~b}$ produces iC $3 \mathrm{~b}, \mathrm{C} 3 \mathrm{f}, \mathrm{C} 3 \mathrm{c}, \mathrm{C} 3 \mathrm{dg}$, $\mathrm{C} 3 \mathrm{~d}$, and $\mathrm{C} 3 \mathrm{~g} .{ }^{20} \mathrm{C} 3$ acts as a point of convergence among the three complement pathways and plays a central role in the process of complement activation. This results in the amplification of the complement response and helps coordinate downstream immune responses. It has become evident that $\mathrm{C} 3$ participates in clearing pathogens and a variety of homeostatic processes, such as tissue regeneration and controlling tumor progression. ${ }^{21}$

Hypocomplementemia was considered to be associated with the consumption of complement components because of complement activation. ${ }^{22}$ The levels of $\mathrm{C} 3$ (not $\mathrm{C} 4$ ) reportedly decreased significantly in patients with septic shock compared to critically ill patients. ${ }^{23}$ Similarly, hypocomplementemia was demonstrated in various viral infections, such as parvovirus B19, Ebola virus, and hepatitis C. ${ }^{24-26} \mathrm{C} 3$ consumption was described in two children with SARSCoV-2-induced Kawasaki-like hyperinflammatory syndrome. In Phase 2 of their illness, both patients developed progressive thrombocytopenia, hepatomegaly, and diffuse edema. Fortunately, they improved after corticosteroid treatment. ${ }^{27}$ Another study reported that very low $\mathrm{C} 3$ levels were observed in a child with pediatric inflammatory multisystem syndrome because of SARS-COV-2 infection. After being treated with high-dose intravenous immunoglobulin and methylprednisolone, the child recovered and the $\mathrm{C} 3$ levels returned to normal. ${ }^{28}$ These studies suggest that complement activation by $\mathrm{C} 3$ hypocomplementemia may be implicated in the pathophysiology of COVID-19.

A retrospective study showed that serum complement C3 levels cannot predict COVID-19 progression. ${ }^{10}$ In contrast, Fang et al reported that decreased complement $\mathrm{C} 3$ was correlated with increased odds of death. ${ }^{8}$ However, in the study by Fang et al, adjustment was only performed for age, sex, laboratory findings, and comorbidities. The lack of adjustment for 
treatment regimens could affect the prognosis of the patients, resulting in a confounding bias. It was reported that plasma levels of complement $\mathrm{C} 3$ are associated with the development of hypertension, ${ }^{29}$ diabetes, ${ }^{30}$ and coronary heart disease. ${ }^{31}$ This may account for the higher comorbidity in the control group with relatively higher $\mathrm{C} 3$ levels. Therefore, we adjusted for confounders using the IPTW method, including age, sex, comorbidities, laboratory tests, and medications on admission. According to the Cox regression analysis with IPTW, there was a significant inverse correlation between C3 hypocomplementemia and clinical worsening in patients with COVID-19. A series of sensitivity analyses also generated supportive evidence. Moreover, our results indicate that the low $\mathrm{C} 3$ population might be experiencing a higher risk of death. No significant associations were observed between the baseline C3 levels and death in the unadjusted and IPTW analyses.

Emerging evidence suggests that complement $\mathrm{C} 3$ activation might be triggered by virus nucleocapsid protein through virus-released pathogen-associated molecular patterns during SARS-CoV-2 infection. ${ }^{32,33}$ Complement and tissue factor (TF)-enriched neutrophil extracellular traps (NETs) are crucial drivers of immunothrombosis. Moreover, C3 blockade can disrupt TF expression in neutrophils, inhibit complement activation, and attenuate platelet-mediated NET-induced thrombogenicity in COVID-19. ${ }^{9}$ Compstatin, a highly selective C3 inhibitor, can inhibit complement activation by binding to $\mathrm{C} 3$ and interfering with the convertase formation and $\mathrm{C} 3$ cleavage. ${ }^{34,35}$ The latest study reported that treatment with the compstatin was safe and successful in a patient with severe acute respiratory distress syndrome (ARDS) following SARS-COV-2 infection. ${ }^{36}$ Another exploratory study in small independent cohorts of severe COVID19 patients indicated that C3 inhibitor (AMY-101) can abrogate COVID-19 hyperinflammation. This results in the decline of CRP and IL-6 levels, improvement of lung function, and resolution of SARS-CoV-2-associated ARDS. ${ }^{37}$ These early clinical results support the evaluation of C3-targeting agents as COVID-19 therapeutics in large prospective trials. The results of our study suggest that the complement system is activated by the consumption of C3 during SARSCoV-2 infection, and COVID-19 patients with C3 hypocomplementemia might benefit from complement inhibition treatment.

There are several limitations to our study. First, this is a single-center observational study with small sample size. Therefore, the results cannot be generalized, and large-scale randomized controlled trials are warranted to assess the associations of C3 hypocomplementemia with clinical worsening and outcomes of COVID-19. Second, all patients with COVID-19 in this designated clinical center were transferred from other hospitals during the outbreak in Wuhan, and the timing of serum C3 level measurement may be inappropriate for indicating the status of complement activation because each patient was at a different stage of the SARS-COV-2 infection on admission. The longitudinal C3 levels could describe the course of complement activation more accurately compared to the values obtained at baseline. According to the dynamic changes in $\mathrm{C} 3$ levels, a reasonable determination can be made regarding the optimal time window for complement intervention. Third, in this designated clinical center, the serum complement C3 level was not routinely measured within $24 \mathrm{~h}$ of admission for every COVID-19 patient. Only patients with C3 levels tested at baseline were considered for this study, which might introduce a certain kind of selection bias.

\section{Conclusion}

In this preliminary study, our results suggest that low serum complement $\mathrm{C} 3$ level is associated with a higher risk for clinical worsening among hospitalized patients with COVID-19. We hypothesize that the C3 level may contribute to the identification of patient populations that could benefit from therapeutic complement inhibition.

\section{Acknowledgments}

We thank all health care workers in the Department of Infectious Diseases, Union Hospital, Tongji Medical College, Huazhong University of Science and Technology in Wuhan. We acknowledge all the medical staff on the front line in Wuhan. We also thank Bullet Edits for its linguistic assistance during the preparation of this manuscript.

\section{Funding}

There is no funding to report. 


\section{Disclosure}

The authors report no conflicts of interest in this work.

\section{References}

1. Wiersinga WJ, Rhodes A, Cheng AC, Peacock SJ, Prescott HC. Pathophysiology, transmission, diagnosis, and treatment of coronavirus disease 2019 (COVID-19): a review. JAMA. 2020;324. doi:10.1001/jama.2020.12839.

2. Nesargikar PN, Spiller B, Chavez R. The complement system: history, pathways, cascade and inhibitors. Eur J Microbiol Immunol. 2012;2:103-111. doi:10.1556/EuJMI.2.2012.2.2

3. Jiang Y, Zhao G, Song N, et al. Blockade of the C5a-C5aR axis alleviates lung damage in hDPP4-transgenic mice infected with MERS-CoV. Emerg Microbes Infect. 2018;7(1):77. doi:10.1038/s41426-018-0063-8

4. Jiang Y, Li J, Teng Y, et al. Complement receptor C5aR1 inhibition reduces pyroptosis in hDPP4-transgenic mice infected with MERS-CoV. Viruses. 2019;11(1):39. doi:10.3390/v11010039

5. Gralinski LE, Sheahan TP, Morrison TE, et al. Complement activation contributes to severe acute respiratory syndrome coronavirus pathogenesis. mBio. 2018;9(5):e01753-18. doi:10.1128/mBio.01753-18

6. Polycarpou A, Howard M, Farrar CA, et al. Rationale for targeting complement in COVID-19. EMBO Mol Med. 2020;12(8):e12642. doi:10.15252/ emmm.202012642

7. Skendros P, Mitsios A, Chrysanthopoulou A, et al. Complement and tissue factor-enriched neutrophil extracellular traps are key drivers in COVID-19 immunothrombosis. J Clin Invest. 2020;130(11):6151-6157. doi:10.1172/JCI141374

8. Fang S, Wang H, Lu L, Jia Y, Xia Z. Decreased complement C3 levels are associated with poor prognosis in patients with COVID-19: a retrospective cohort study. Int Immunopharmacol. 2020;89(Pt A):107070. doi:10.1016/j.intimp.2020.107070

9. Qin C, Zhou L, Hu Z, et al. Dysregulation of immune response in patients with coronavirus 2019 (COVID-19) in Wuhan, China. Clin Infect Dis. 2020;71(15):762-768. doi:10.1093/cid/ciaa248

10. Zhang Z, Li X, Zhang W, Shi ZL, Zheng Z, Wang T. Clinical features and treatment of 2019-nCov pneumonia patients in Wuhan: report of a couple cases. Virol Sin. 2020;35(3):330-336. doi:10.1007/s12250-020-00203-8

11. China NHC. Guidelines of the diagnosis and treatment of novel coronavirus pneumonia (trial version 8). in Chinese. Available from: http://www. nhc.gov.cn/yzygj/s7653p/202008/0a7bdf12bd4b46e5bd28ca7f9a7f5e5a.shtml. Accessed January 15, 2022

12. Birmingham DJ, Irshaid F, Nagaraja HN, et al. The complex nature of serum C3 and C4 as biomarkers of lupus renal flare. Lupus. 2010;19 (11):1272-1280. doi:10.1177/0961203310371154

13. Complement component 3 (C3) [website on the Internet]. UCSF Benioff Children's Hospitals; 2022. Available from: https://www.ucsfbenioffchil drens.org/medical-tests/003539. Accessed January 15, 2022.

14. How to Read Complement C3 Test Report [website on the Internet]. MediFee.com; 2022. Available from: https://www.medifee.com/how-to-read /complement-c3-test-report. Accessed January 15, 2022.

15. C3 And C4 Blood Test [website on the Internet]. PORTEA - HEAL AT HOME; 2022. Available from: https://www.portea.com/labs/diagnostictests/c3-and-c4-blood-test-204/. Accessed January 15, 2022.

16. Grein J, Ohmagari N, Shin D, et al. Compassionate use of remdesivir for patients with severe COVID-19. N Engl J Med. 2020;382(24):2327-2336. doi:10.1056/NEJMoa2007016

17. Stuart EA, Lee BK, Leacy FP. Prognostic score-based balance measures can be a useful diagnostic for propensity score methods in comparative effectiveness research. J Clin Epidemiol. 2013;66(8 Suppl):S84-S90.e1. doi:10.1016/j.jclinepi.2013.01.013

18. West EE, Kolev M, Kemper C. Complement and the regulation of T cell responses. Annu Rev Immunol. 2018;36:309-338. doi:10.1146/annurevimmunol-042617-053245

19. Nasir A, Caetano-Anolles G. A phylogenomic data-driven exploration of viral origins and evolution. Sci Adv. 2015;1(8):e1500527. doi:10.1126/ sciadv. 1500527

20. Janssen BJ, Huizinga EG, Raaijmakers HC, et al. Structures of complement component C3 provide insights into the function and evolution of immunity. Nature. 2005;437(7058):505-511. doi:10.1038/nature04005

21. Ricklin D, Reis ES, Mastellos DC, Gros P, Lambris JD. Complement component C3 - the "Swiss Army Knife" of innate immunity and host defense. Immunol Rev. 2016;274(1):33-58. doi:10.1111/imr.12500

22. Atsumi T, Amengual O, Koike T. Antiphospholipid syndrome: pathogenesis. In: Systemic Lupus Erythematosus. Fifth ed. Academic Press; 2011:945-965.

23. Lin RY, Astiz ME, Saxon JC, Saha DC, Rackow EC. Alterations in C3, C4, factor B, and related metabolites in septic shock. Clin Immunol Immunopathol. 1993;69(2):136-142. doi:10.1006/clin.1993.1161

24. Hashizume H, Kageyama R. Hypocomplementemia is a diagnostic clue for parvovirus B19 infection in adults. J Dermatol. $2017 ; 44(3)$ :e27. doi:10.1111/1346-8138.13469

25. Brudner M, Karpel M, Lear C, et al. Lectin-dependent enhancement of Ebola virus infection via soluble and transmembrane C-type lectin receptors. PLoS One. 2013;8:e60838. doi:10.1371/journal.pone.0060838

26. Itoh K, Tanaka H, Shiga J, et al. Hypocomplementemia associated with hepatitis C viremia in sera from voluntary blood donors. Am J Gastroenterol. 1994;89(11):2019-2024.

27. Licciardi F, Pruccoli G, Denina M, et al. SARS-CoV-2-induced Kawasaki-like hyperinflammatory syndrome: a novel COVID phenotype in children. Pediatrics. 2020;146(2):e20201711. doi:10.1542/peds.2020-1711

28. Regev T, Antebi M, Eytan D, et al. Pediatric inflammatory multisystem syndrome with central nervous system involvement and hypocomplementemia following SARS-COV-2 infection. Pediatr Infect Dis J. 2020;39(8):e206-e207. doi:10.1097/INF.0000000000002804

29. Engström G, Hedblad B, Berglund G, Janzon L, Lindgärde F. Plasma levels of complement C3 is associated with development of hypertension: a longitudinal cohort study. J Hum Hypertens. 2007;21(4):276-282. doi:10.1038/sj.jhh.1002129

30. Engström G, Hedblad B, Eriksson KF, Janzon L, Lindgärde F. Complement C3 is a risk factor for the development of diabetes: a population-based cohort study. Diabetes. 2005;54(2):570-575. doi:10.2337/diabetes.54.2.570 
31. Engström G, Hedblad B, Janzon L, Lindgärde F. Complement C3 and C4 in plasma and incidence of myocardial infarction and stroke: a population-based cohort study. Eur J Cardiovasc PrevRehabil. 2007;14(3):392-397. doi:10.1097/01.hjr.0000244582.30421.b2

32. Risitano AM, Mastellos DC, Huber-Lang M, et al. Complement as a target in COVID-19? Nat Rev Immunol. 2020;20(6):343-344. doi:10.1038/ s41577-020-0320-7

33. Gao T, Hu MD, Zhang XP, Li HZ, Cao C. Highly pathogenic coronavirus N protein aggravates lung injury by MASP-2-mediated complement over-activation. medRxiv. 2020. doi:10.1101/2020.03.29.20041962

34. Mastellos DC, Yancopoulou D, Kokkinos P, et al. Compstatin: a C3-targeted complement inhibitor reaching its prime for bedside intervention. Eur $J$ Clin Invest. 2015;45(4):423-440. doi:10.1111/eci.12419

35. Mastellos DC, Ricklin D, Lambris JD. Clinical promise of next-generation complement therapeutics. Nat Rev Drug Discov. 2018;18:707-729. doi:10.1038/s41573-019-0031-6

36. Mastaglio S, Ruggeri A, Risitano AM, et al. The first case of COVID-19 treated with the complement C3 inhibitor AMY-101. Clin Immunol. 2020;215:108450. doi:10.1016/j.clim.2020.108450

37. Mastellos DC, Pires da Silva BGP, Fonseca BAL, et al. Complement C3 vs C5 inhibition in severe COVID-19: early clinical findings reveal differential biological efficacy. Clin Immunol. 2020;220:108598. doi:10.1016/j.clim.2020.108598

International Journal of General Medicine

Dovepress

\section{Publish your work in this journal}

The International Journal of General Medicine is an international, peer-reviewed open-access journal that focuses on general and internal medicine, pathogenesis, epidemiology, diagnosis, monitoring and treatment protocols. The journal is characterized by the rapid reporting of reviews, original research and clinical studies across all disease areas. The manuscript management system is completely online and includes a very quick and fair peer-review system, which is all easy to use. Visit http://www.dovepress.com/testimonials.php to read real quotes from published authors.

Submit your manuscript here: https://www.dovepress.com/international-journal-of-general-medicine-journal 